BMJ Open Sport \& Exercise Medicine

\section{Effect of Tai Chi on muscle strength, physical endurance, postural balance and flexibility: a systematic review and meta-analysis}

To cite: Wehner C, Blank C, Arvandi M, et al. Effect of Tai Chi on muscle strength, physical endurance, postural balance and flexibility: a systematic review and meta-analysis. BMJ Open Sport \& Exercise Medicine 2021;7:e000817. doi:10.1136/ bmjsem-2020-000817

- Additional material is published online only. To view, please visit the journal online (http://dx.doi.org/10.1136/ bmjsem-2020-000817).

Accepted 25 January 2021

Check for updates

(c) Author(s) (or their employer(s)) 2021. Re-use permitted under CC BY-NC. No commercial re-use. See rights and permissions. Published by BMJ.

${ }^{1}$ Institute for Sports Medicine, Alpine Medicine and Health Tourism, Private University for Health Sciences Medical Informatics and Technology, Hall, Austria

${ }^{2}$ Institute of Public Health, Medical Decision Making and HTA, Private University for Health Sciences Medical Informatics and Technology, Hall, Austria ${ }^{3}$ WuYuan - School of Chinese Martial Arts, Kungfu, Taijiquan and Qigong, Munich, Germany

Correspondence to Christian Wehner; christian.wehner@edu.umit.at

\section{ABSTRACT}

Objective To investigate the impact of Tai Chi training on muscle strength, physical endurance, postural balance and flexibility, as measured by tests commonly used in healthrelated fitness or competitive sports contexts.

Design Systematic review and meta-analysis.

Data sources The following databases were searched up to 31 July 2020: CINAHL, Cochrane Library, MEDLINE via PubMed and SPORTDiscus.

Eligibility criteria for studies Inclusion: (1)

Randomised controlled trials published in German or English; (2) Tai Chi used as an intervention to improve physical performance; (3) Test methods commonly used in health-related fitness or competitive sports and (4) Participants aged $\geq 16$ years (irrespective of health status). Exclusion: (1) Studies not focusing on Tai Chi or including Tai Chi mixed with other interventions and (2) Modified or less than eight Tai Chi movements.

Results Out of 3817 records, 31 studies were included in the review, 21 of them in the meta-analysis. Significant improvements in handgrip strength $(2.34 \mathrm{~kg}, 95 \% \mathrm{Cl} 1.53$ to 3.14$)$, walking distance during $6 \mathrm{~min}(43.37 \mathrm{~m}, 95 \% \mathrm{Cl}$ 29.12 to 57.63$)$, standing time in single-leg-stance with open eyes $(6.41 \mathrm{~s}, 95 \% \mathrm{Cl} 4.58$ to 8.24$)$ and thoracolumbar spine flexibility $(2.33 \mathrm{~cm}, 95 \% \mathrm{Cl} 0.11$ to 4.55$)$ were observed.

Conclusion Tai Chi training seems to moderately improve physical fitness when evaluated by tests used in health-related fitness or competitive sports. Moreover, thoracolumbar spine flexibility seems to be a factor in the improvement of postural balance. Further research is needed, including younger healthy participants performing a widely used, standardised form (eg, Peking-style routine) with high-intensity movements (eg, use of lower stances).

\section{INTRODUCTION}

Tai Chi Quan, also often called Tai Chi, is one style of the Chinese martial arts (Wushu) and consists of defined motion sequences performed with and without weapons (eg, swords), as well as routines performed with partners. There are five traditional styles of Tai Chi: Chen, Yang, Wu Yuxiang, Wu and Sun style. The most famous routine in the

\section{Summary box}

What is already known?

- Tai Chi training has positive effects on a variety of chronic diseases (eg, osteoarthritis) and healthrelated issues (eg, reduced risk of falling).

- Tai Chi training exerts a positive impact not only on physical parameters, but also on mental health.

- There is good evidence for positive effects of Tai Chi training for older people and patient populations, as most previous studies concentrated on these populations.

\section{What are the new findings?}

- There is evidence that Tai Chi training can also moderately improve physical fitness as measured by tests commonly applied in health-related fitness or competitive sports contexts; for healthy people such tests are more relevant compared with the clinical assessment tools used for unfit and patient populations. Improvements were observed in handgrip strength, functional capacity, postural balance and thoracolumbar flexibility.

- We hypothesise that not only slow motions of the legs and kicking movements while standing on one leg, which are characteristic in Tai Chi but also the improvement of thoracolumbar flexibility enhance postural balance.

field of health sports, which was published in the 1950s, is the so-called Peking style routine, which features 24 movements based on the Yang style. ${ }^{1}$

In the past few decades, a considerable number of studies, systematic reviews and meta-analyses concerning Tai Chi have been published. They mostly focused on the healthrelated consequences of Tai Chi training, and either investigated specific groups of of individuals with chronic diseases, for example, Parkinson's disease, ${ }^{2}$ or specific health-related issues, for example, risk of falling. ${ }^{3}$

Positive effects of Tai Chi training were reported for cancer, ${ }^{4}$ osteoarthritis, ${ }^{4}$ heart failure ${ }^{4}$ chronic obstructive pulmonary 
disease (COPD),${ }^{45}$ type 2 diabetes mellitus, ${ }^{6}$ coronary heart disease, ${ }^{7}$ chronic lower back pain ${ }^{8-10}$ and Parkinson's disease. ${ }^{2}$ Similar observations were summarised in the systematic review entitled 'Evidence Map of Tai Chi' by Hempel et al, ${ }^{11}$ which included 107 systematic reviews. They identified 40 different health-related research fields, classified as 'evidence of no effect', 'unclear evidence' or 'evidence of a potential positive effect'. The first research field, with eight subareas, dealt with global health issues, for example, general health, psychological well-being and the health of older adults. In two out of these eight subareas, which focused on cognitive performance and balance confidence, the authors found a potential positive effect of Tai Chi. For life participation, another subarea, there was no effect of Tai Chi, while for the remaining subareas the evidence was unclear. The second and largest research field, comprising 29 subareas, pertained to the impact of Tai Chi on individuals with chronic diseases. Potential benefits were noted for hypertension, COPD, osteoarthritis, pain and depression. ${ }^{11}$ In contrast to the more recent analysis of Zhou et $a l,{ }^{6}$ no improvement of biochemical or psychological parameters was seen in patients with type 2 diabetes. For the remaining 23 subareas, the evidence was unclear. The smallest research field, comprising only 3 subareas out of $40,{ }^{11}$ is directly related to muscle strength, physical endurance, speed, coordination and flexibility, which are crucial for learning and executing sportive motion sequences. ${ }^{12}$ Findings for these subareas varied, including no effects (aerobic capacity), potential positive effects (muscle strength) and unclear evidence (balance general).

A large majority of the randomised controlled trials (RCTs) included in the existing systematic reviews and meta-analyses dealing with Tai Chi used questionnaires (eg, the 36-item Short-Form (SF-36) for quality of life ${ }^{13}$ ) or clinical assessment tests for individuals with chronic diseases. Clinical assessment tests like the Timed Up and Go test for the evaluation of functional mobility ${ }^{14}$ are performed to detect, diagnose or monitor diseases, or to determine a course of treatment. Some RCTs analysed biomedical parameters (eg, inflammatory markers ${ }^{15}$ ). A small proportion of the RCTs used tests that were performed in health related fitness or competitive sports to evaluate global or specific sports abilities or skills. These tests are primarily used for healthy and fit people, but can also be used for individuals with injuries or chronic diseases, and are often part of a larger test battery (eg, single-leg-stance (SLS) test with closed eyes as part of the 'Eurofit for adults' ${ }^{16}$ ).

Numerous studies and reviews have investigated the impact of Tai Chi training on global health issues and chronic diseases, primarily using clinical assessment tools. However, the influence of Tai Chi training, evaluated only by tests commonly applied in health related fitness or competitive sports, has not yet been investigated to the best of our knowledge. Thus, the goal of this review and meta-analysis is to summarise results of existing studies to determine whether Tai Chi training can significantly improve muscle strength, physical endurance, functional capacity, postural balance and flexibility in healthy persons and individuals with chronic diseases only by applying tests that are used in health-related fitness or competitive sports contexts.

\section{METHODS}

To address the research question outlined above, a systematic review and meta-analysis was performed. The reportage for this systematic review is based on the Preferred Reporting Items for Systematic Reviews and Meta-Analyses Statement. ${ }^{17}$

\section{Search strategy}

The following databases were searched up to 31 July 2020: CINAHL, the Cochrane Library, MEDLINE and SPORTDiscus. Search terms included controlled vocabulary queries (eg, MeSH Terms), as well as specific text words in titles and abstracts. The main keyword for the database searches was "Tai Chi" (and its synonymous spellings: "Tai-Chi”, "T’ai Chi”, "Taiji”, "Tai Ji”, "Tai-ji" and "Taijiquan"). When screening for fundamental attributes, broad terms ("physical exercise", "physical fitness", "physical effects" and "motor function"), and specialised terms ("strength", "muscle strength", "muscular strength", "strength training", "aerobic exercise", "aerobic physical exercise", "6-Minute Walk Test", "endurance", "aerobic capacity", "cardiorespiratory fitness", "aerobic strengthening", "coordination", "balance ability", "tandem stance", "single leg stance", "tandem standing test", "kinesthetic sense", "kinaesthesia", "proprioception exercise", "flexibility", "mobility" and "stiffness") were used. These terms were systematically combined using Boolean operators ("AND", "OR"). A handsearch was performed by screening the reference sections of included articles.

\section{Inclusion and exclusion criteria}

RCTs published in German or English were included if they fulfilled the following criteria: investigated Tai Chi as an intervention to improve muscle strength, physical endurance, functional capacity, postural balance or flexibility; used test methods that are prevalent in sports science and typically employ physical units (eg, newton, time in second/minute); included participants aged 16 years and above irrespective of their health status and any duration of intervention. Studies not focusing on Tai Chi were excluded, as were those including Tai Chi mixed with other interventions, modified Tai Chi movements or less than 8/10 (without/with starting/closing movements) Tai Chi movements.

\section{Data collection}

Titles and abstracts were screened to assess study eligibility. For all eligible studies, full texts were retrieved. Two reviewers assessed the full texts in detail with regard to the eligibility criteria pertaining to Tai Chi style, Tai Chi movements, intervention protocol, randomisation 


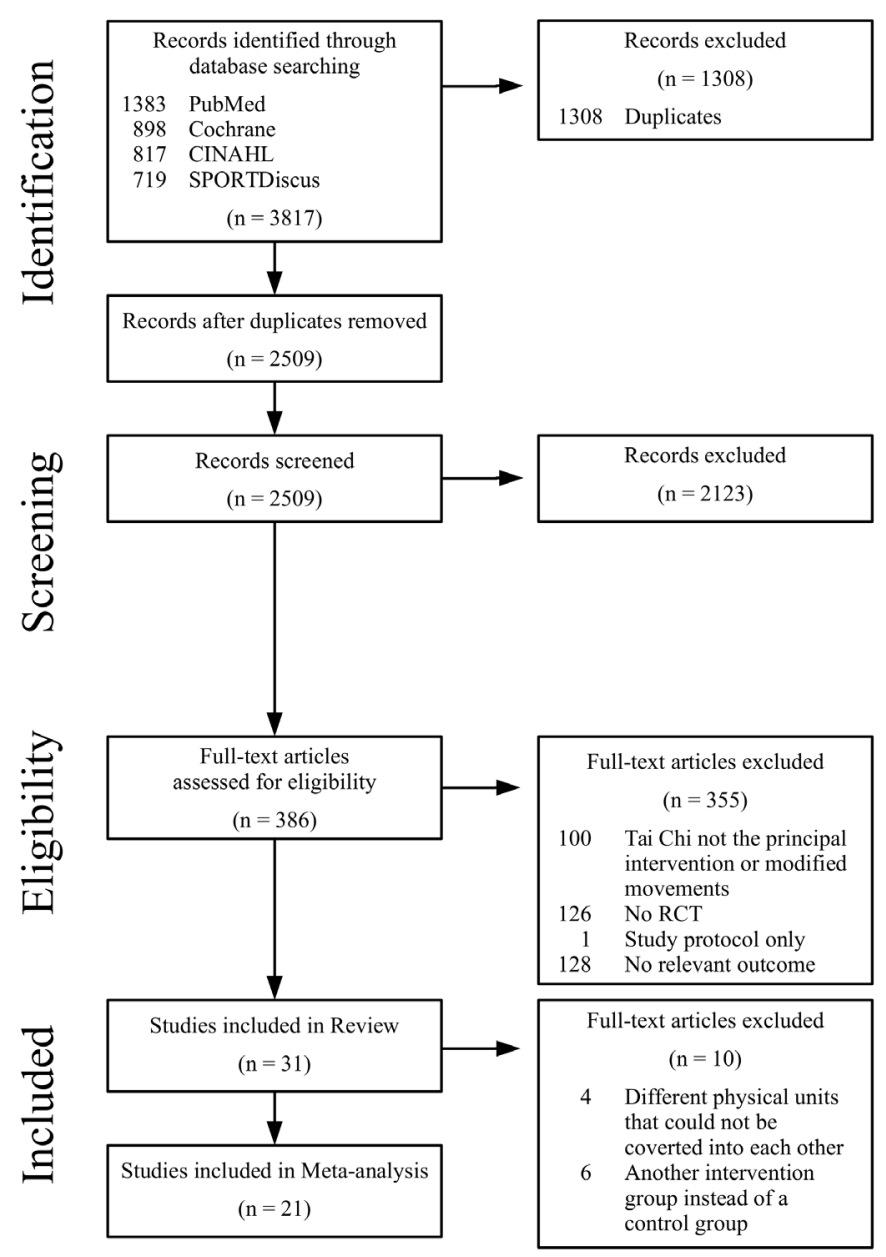

Figure 1 Flow diagram based on the PRISMA statement. ${ }^{17}$ PRISMA, Preferred Reporting Items for Systematic Reviews and Meta-Analyses; RCT, randomised controlled trial.

process and testing procedures. Data were entered into the Cochrane data collection form for intervention reviews $^{18}$ and verified by the second reviewer. Disagreements were resolved by discussion. Furthermore, study quality and risk of bias (eg, random sequence generation, allocation concealment, blinding, incomplete outcome data, selective outcome reporting and other biases) were assessed. ${ }^{18}$ The whole study selection process is shown in figure 1 .

\section{Data items}

Study type and design, participant characteristics (age, sex and diagnosed diseases if available), intervention characteristics (duration of participation, frequency and duration of sessions, description of intervention and control arms), outcome measures and results (mean, SD, SE, CI, preintervention and postintervention differences within and between groups, significance level and units of measurement) and formal details (ethical approval, informed consent and funding sources) were extracted from the studies.

Outcome data were classified into (1) muscle strength (physical units: newton, newton metre, $\mathrm{Nm} / \mathrm{kg}$ body weight, kilogram, $\mathrm{kg} / \mathrm{kg}$ body weight), including handgrip, elbow extensor, elbow flexor, dorsiflexor, plantar flexor, knee extensor, knee flexor and hip flexor strength, (2) physical endurance respectively functional capacity, including incremental shuttle walk test (ISWT; physical unit: metre), endurance shuttle walk test (ESWT; physical unit: second), maximal oxygen consumption (VO2max; physical unit: $\mathrm{ml} / \mathrm{kg} / \mathrm{min}$ ), 6 min walk test (6MWT; physical units: metre, mile, yard), 12 min walk test (12MWT; physical unit: mile), (3) postural balance (physical unit: second), including SLS with open eyes, SLS with closed eyes, tandem stance (TDS) with open eyes, TDS with closed eyes and other, and (4) flexibility (physical units: centimetre, inch), including stand-andreach test, sit-and-reach test, shoulder range of motion and hip range of motion. Details are displayed in online supplemental table 1 .

\section{Data synthesis}

Outcomes that were reported by a sufficient number of studies were included in a meta-analysis. All other outcomes are descriptively summarised in a table.

\section{Meta-analysis}

Out of 31 studies, four with different (and thus noncomparable) physical units ${ }^{19-22}$ and six without control groups $^{13}$ 23-27 were excluded. Outcomes reported by a sufficient number of studies were included in the metaanalysis. All other outcomes that are not used in the meta-analysis are descriptively summarised in online supplemental table 2 .

A meta-analysis was performed on handgrip strength (5 studies), ${ }^{28-32}$ knee extensor muscle strength (6 studies), ${ }^{32-37}$ functional capacity (4 studies), ${ }^{1428} 3839$ SLS with eyes open (10 studies) ${ }^{14293240-46}$ and thoracolumbar spine flexibility (4 studies). ${ }^{3645-47}$ For the analysis of knee extensor muscle strength, the pooled standardised mean difference based on Hedges' g was calculated because three different physical units were used (newton, newton metre and kilogram). To ensure comparability between the studies, for studies that distinguished between men and women ${ }^{32}$ and between left and right leg, ${ }^{29} 374042$ overall mean values were calculated for comparison with the studies providing only average values. Using the statistical software Stata (V.15.1; StataCorp), pooled mean effects and 95\% CIs were calculated for all included studies. A random-effects model was used due to the presumably high degree of heterogeneity, and to ensure a more balanced weighting when including the smaller studies. In addition, a leave-one-out cross-validation was performed. Heterogeneity was examined by Cochran's $Q$ test. Subsequently, the observed heterogeneity was quantified by $\mathrm{I}^{2}$ and a univariate meta-regression analysis was performed to investigate three variables, which can be considered as possible causes when study population (high percentage of older people across all studies) and study design are taken into account. These variables are (1) different numbers of Tai Chi movements, (2) presence of a chronic disease (binary variable: yes/no) and 
(3) existing balance impairment (binary variable: yes/ no). The latter was investigated in four studies including only balanced impaired individuals. ${ }^{23} 244046$ For all five outcomes, a forest plot was generated.

\section{RESULTS}

Through database searching, a total of 3817 records were identified. After removing 1308 duplicates, 2509 records were screened for title and abstract. In total, fulltext articles of 386 records were retrieved and assessed for eligibility (refer to figure 1). Based on the inclusion and exclusion criteria, further articles were eliminated, resulting in 31 articles being included in the review. After excluding another ten articles due to the above stated reasons, 21 out of the 31 articles were included in the meta-analysis. Handsearch revealed no additional studies.

\section{Study characteristics}

The characteristics of all included studies are summarised in the following section and are listed in online supplemental table 1 .

The majority of the studies (23 out of 31 ) were twoarmed RCTs and the remaining (eight) studies used a three-armed RCT design. ${ }^{19} 21242731324445$ Out of the twoarmed RCTs, 19 studies comprised a Tai Chi intervention group and a control group that did not perform physical exercises, three studies had a control group performing physical excercises ${ }^{132325}$ and one study compared two intervention groups, each with a different Tai Chi style. ${ }^{26}$ One of the three-armed RCTs also compared three groups with different Tai Chi styles. ${ }^{27}$

The number of participants ranged from $14^{25}$ to $368 .{ }^{23}$ In eight studies, only women were included. ${ }^{1920222528343644}$ The participants were mostly healthy people aged 60 years or more, although in one study the participants were aged between 16 and 25 years. ${ }^{47}$ Four studies investigated people with history ${ }^{2324}$ or higher risk of falling (definded by either Berg balance score $\leq 45^{40}$ or one leg standing time between 5 and $20 \mathrm{~s}^{46}$ ), while 11 studies examined individuals with the following chronic diseases: Parkinson disease, ${ }^{14}$ osteopaenia, ${ }^{20}$ osteoarthritis,,${ }^{135}{ }^{41}$ rheumatoid arthritis, ${ }^{30}$ fibromyalgia, ${ }^{36}{ }^{38} \mathrm{COPD}^{37} 39$ and breast cancer survivors. ${ }^{28}$

The intervention duration ranged from 3 weeks $^{40}$ to 12 months ${ }^{32}$ and the training frequency varied from one to seven times per week (40-90 min per training session). Half of the studies used a routine comprising 24 movements based on the Yang style. The other studies used fewer movements, except for two that comprised $30,{ }^{14}$ $42^{27}$ and $56^{27}$ movements respectively. Only three studies included the Chen, ${ }^{24} \mathrm{Wu}^{35}$ or $\mathrm{Sun}^{37}$ style. In addition, two studies compared two or more styles. ${ }^{26} 27$

The outcome measures were as follows. The strength of different muscles was assessed in 14 studies, among which seven investigated handgrip strength, ${ }^{19} 232829313238$ one the biceps and triceps muscles, ${ }^{25}$ three the plantiflexor and dorsiflexor muscles, ${ }^{213335}$ four the knee flexor muscles, ${ }^{2533-35}$ eight the knee extensor muscles ${ }^{192532-37}$ and one the hip flexor muscles. ${ }^{35}$ Physical endurance was assessed in three, ${ }^{19} 2537$ functional capacity in six studies using the 6MWT 1314283839 or the $12 \mathrm{MWT} \mathrm{T}^{31}$ Balance ability was assessed in 14 studies. The most common test, which was used in 12 studies, was the SLS with open eyes. ${ }^{14} 1924293240-46$ SLS with closed eyes was used in six studies, ${ }^{19} 262742-44$ while tandem stance with open eyes was used in three studies, ${ }^{14} 3240$ and with closed eyes in only one study. ${ }^{43}$ Flexibility was the most infrequently investigated parameter. Only a few studies assessed the flexibility of the limb joints. One study examined the shoulder, ${ }^{28}$ two the hip flexibility ${ }^{19} 22$ and one plantar flexion of the foot. ${ }^{22}$ The thoracolumbar spine flexibility was assessed by five studies, using either the sit-and-reach ${ }^{19} 364547$ or standand-reach test. ${ }^{46}$

\section{Summary of results}

The outcomes in the Tai Chi intervention groups can be divided into four categories (muscular strength, physical endurance/functional capacity, postural balance and flexibility), as described below.

\section{Muscular strength}

Regarding handgrip strength, three ${ }^{192328}$ out of seven studies demonstrated a significant improvement, while one study ${ }^{31}$ reported a decrease. Knee flexor strength was significantly improved in two studies. ${ }^{25}{ }^{33}$ Knee extensor muscle strength increased significantly in four studies. ${ }^{19} 253637$ No significant differences were found with respect to the strength of the biceps and triceps muscles, plantiflexor and dorsiflexor muscles, or hip flexor muscles.

\section{Physical endurance/functional capacity}

In total, three studies investigated physical endurance. One study ${ }^{19}$ found a significant increase of VO2max with Tai Chi training, whereas another study ${ }^{25}$ observed no change. In the case of the ISWT $/ \mathrm{ESWT},{ }^{37}$ a significant increase with Tai Chi training was reported. In terms of functional capacity, as measured by walking distance using the 6MWT, four studies, ${ }^{1328} 3839$ demonstrated a significant improvement with Tai Chi training. In contrast, one study ${ }^{14}$ found no change and Wolf $e t a l^{31}$ reported a significant decrease for the 12MWT.

\section{Postural balance}

The test most commonly used (12 studies) to assess the balance of the participants was the SLS with open eyes. An improvement in balance was observed by seven studies. ${ }^{24} 4042-46$ The SLS test with closed eyes was used in six studies, of which three ${ }^{194244}$ reported a significant increase in standing time. Regarding the TDS test with open eyes, as used in three studies, ${ }^{14} 3240$ a significantly prolonged standing time was noted in one study, ${ }^{40}$ while in the one study that used the TDS test with closed eyes, no significant change was reported. ${ }^{43}$

\section{Flexibility}

Flexibility was assessed by seven studies. A significant improvement of shoulder mobility was found in one study. ${ }^{28}$ One study ${ }^{22}$ observed a significant improvement 
in hip flexor range and plantar flexion of the foot, whereas another study ${ }^{19}$ found no change. No significant improvement was found in knee range of motion. ${ }^{13}$ Out of five studies that evaluated thoracolumbar spine flexibility, four ${ }^{19} 364647$ demonstrated a significant improvement with Tai Chi training.

\section{Risk of bias within studies}

The results of the risk of bias assessment are shown in online supplemental table 3. In six studies, ${ }^{24} 2533344345$ randomisation methods and allocation concealment were not specified, while three studies ${ }^{27} 3140$ did not report use of allocation concealment. Due to the nature of the intervention, blinding of the participants and instructors was not possible in those studies. In total, 17 studies reported blinding of the outcome assessors, 13 did not specify and 1 study ${ }^{26}$ stated that the assessors were not blinded to group allocation. One study ${ }^{31}$ reported the baseline characteristics of the participants, but not those of the analysed participants after drop outs. In addition, no adjustment for multiple comparisons was performed in that study. ${ }^{31}$

\section{Meta-analysis \\ Results}

The results of the random-effects model meta-analysis with pooled mean effects, $95 \%$ CIs and study weights are presented as forest plots in figures 2 and 3. Regarding handgrip strength (figure 2A), a significant pooled mean improvement of $2.34 \mathrm{~kg}$ (95\% CI 1.53 to 3.14 ) was observed. The pooled standardised mean difference based on Hedges' g showed no significant effect $(-0.18,95 \%$ CI -0.10 to 0.47$)$ on knee extensor muscle strength (figure 2B). In the case of functional capacity, as measured by the 6MWT (figure 3A), a significant increase in walking distance $(43.37 \mathrm{~m}, 95 \%$ CI 29.12 to 57.63$)$ was found. For the SLS test with open eyes (figure 3B), a significant increase in testing time of $6.41 \mathrm{~s}(95 \% \mathrm{CI}$ 4.58 to 8.24 ) was noted. Thoracolumbar spine flexibility (figure 3C) showed a significant improvement of $2.33 \mathrm{~cm}$ (95\% CI 0.11 to 4.55 ).

\section{Heterogeneity and publication bias}

The $\mathrm{I}^{2}$ of the studies of the different outcomes ranged between $84.4 \%$ and $100 \%$, indicating a considerable between study heterogenity. The univariate meta-regression analysis, which was used to investigate the influence of three explanatory variables on the observed heterogeneity, showed no influence on the two variables presence of a chronic disease and different numbers of Tai Chi movements. In the case of thoracolumbar spine flexibility, the results of the univariate meta-regression analysis showed that $91.4 \% \quad(p=0.029)$ of the between study heterogeneity could be explained by the explanatory variable balance impairment. The consistency of the leave-one-out cross-validation showed that the results of the meta-analyses are robust.

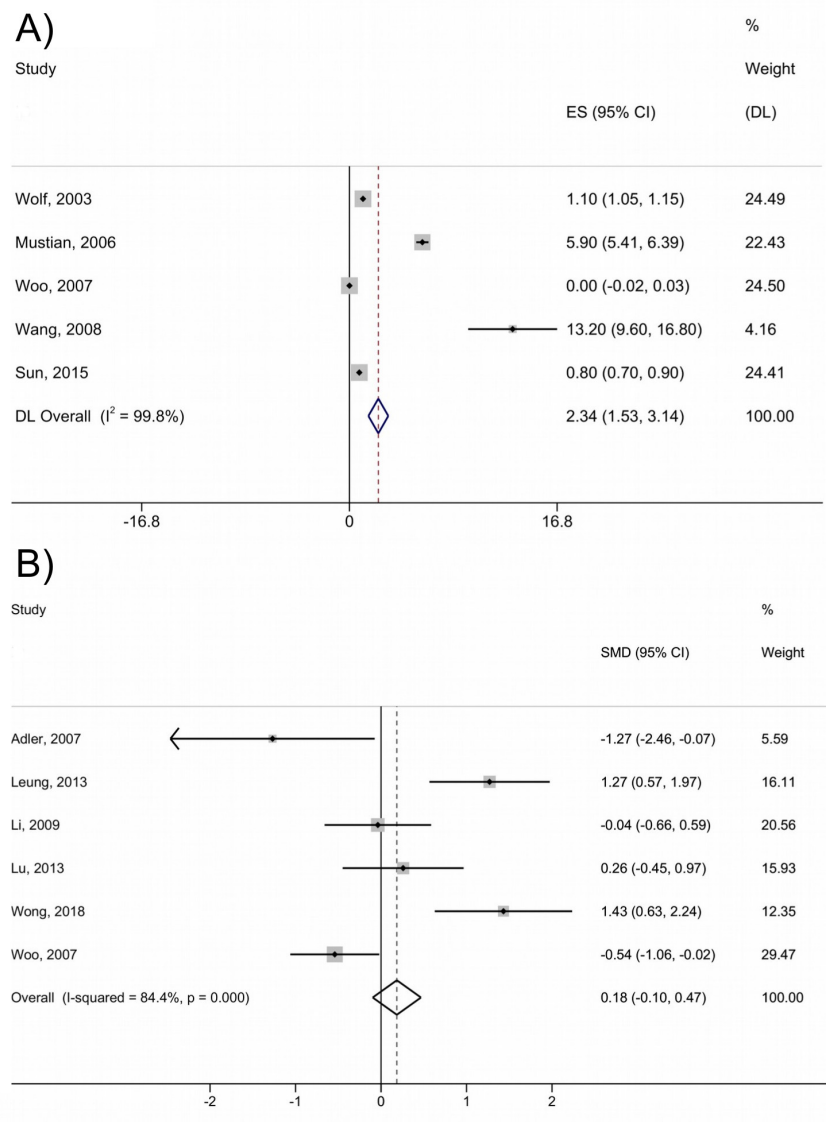

Figure 2 Forest plot from a random-effects meta-analysis of mean changes $(95 \% \mathrm{Cl})$ in $(\mathrm{A})$ handgrip strength $(\mathrm{kg})$ and (B) knee extensor muscle strength (standardised mean difference (SMD) based on Hedges' g). DL, DerSimonianLaird estimator; ES, effect size.

According to the Cochrane Handbook for Systematic Reviews, ${ }^{48}$ at least 10 included studies are needed to test for funnel plot asymmetry. Therefore, sufficient data only for the SLS test with open eyes was available for the analysis. The funnel plot in figure 4 shows an almost symmetric distribution $(\mathrm{p}=0.657)$, which suggests that there is no publication bias for this outcome. All other outcomes included considerably fewer studies, so no test for funnel plot asymmetry was applied.

\section{DISCUSSION}

The purpose of this systematic review and meta-analysis was to investigate whether Tai Chi training could improve muscle strength, physical endurance, postural balance and flexibility in healthy persons and individuals with chronic diseases, when measuring these parameters only with physical performance tests commonly used in health-related fitness or competitive sports contexts. The meta-analysis showed significant improvements in handgrip strength, functional capacity (measured by the 6MWT), postural balance (measured by SLS test with open eyes) and thoracolumbar spine flexibility with Tai Chi training. No significant effect was found in knee extensor muscle strength. 
A)

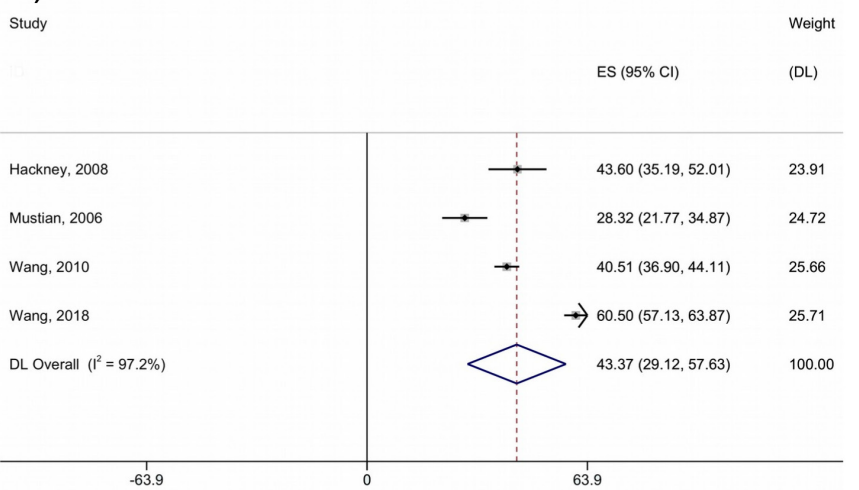

B)

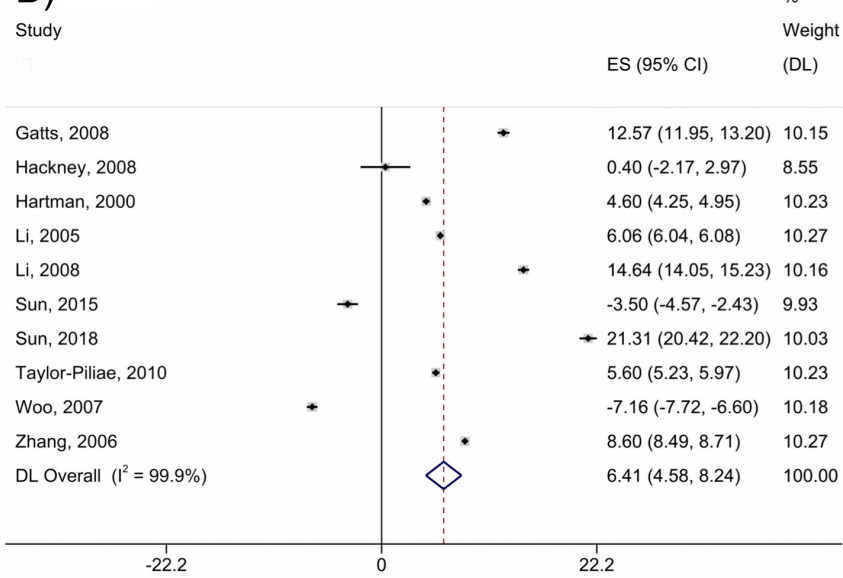

C)

study

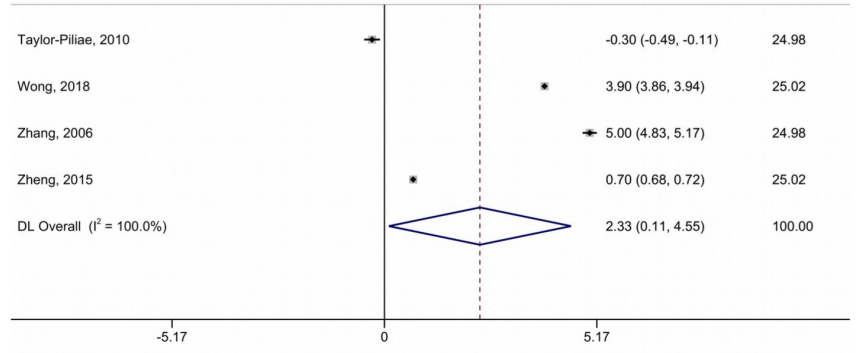

Figure 3 Forest plot from a random-effects meta-analysis of mean changes $(95 \% \mathrm{Cl})$ in $(\mathrm{A})$ functional capacity (measured by walking distance in $6 \mathrm{~min}$ ) (m), (B) singleleg-stance with eyes open (s) and $(\mathrm{C})$ thoracolumbar spine flexibility (cm). DL, DerSimonian-Laird estimator; ES, effect size.

The significant improvement of $2.34 \mathrm{~kg}$ in handgrip strength, also supported by Hwang $e t a l^{23}$ is only a slight effect, which is below the minimal clinically important difference (MCID) of 5.0-6.5 kg stated by Bohannon. ${ }^{49}$ Thus, this improvement should not be seen as an increase in strength, but rather in its function as predictor of total muscle strength in children and young people, ${ }^{50}$ and of daily functioning in the oldest. ${ }^{51}$ Therefore, the

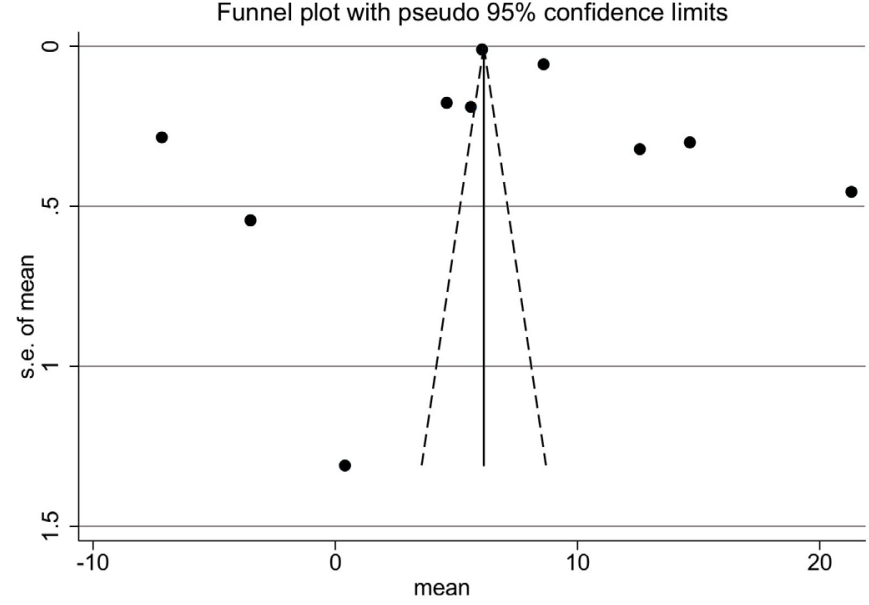

Figure 4 Funnel plot of the studies that investigated single leg stance with eyes open.

improvement in handgrip strength noted in this metaanalysis, suggests that Tai Chi training has a moderate beneficial effect for improving the physical health of people of any age.

In the case of functional capacity, the meta-analysis, which included only the 6MWT walking distance, revealed a significant improvement in walking distance by $43,37 \mathrm{~m}$. According to Bohannon and Crouch, ${ }^{52}$ who reported for the MCID of the 6MWT a range between $14.0 \mathrm{~m}$ and $30.5 \mathrm{~m}$, the improvement of $43.37 \mathrm{~m}$ walking distance can be considered as clinically relevant. In contrast to our findings, Wolf et $a l^{31}$ who used the 12MWT, observed a significant decrease in walking distance by 0.02 miles. One possible reason for this discrepancy could be the participants' fitness status. While the participants in the 6MWT groups were all individuals with chronic diseases, Wolf $e t a l^{31}$ tested only healthy participants on the 12MWT. It is possible that the exercise intensity of Tai Chi, classified by Ainsworth et $a \bar{l}^{\tilde{3}}$ as moderate intensity with four metabolic equivalents, is efficacious for improving exercise performance only in persons suffering from chronic diseases, and not in healthy people with a relatively high baseline level of fitness. In contrast, Audette $e t a l^{19}$ reported a significant increase in VO2max, indicative of better aerobic fitness, in older healthy women after Tai Chi training. However, VO2max was not measured directly, instead being calculated based on the submaximal ergometry results. In addition, it is also possible that the intensity of the performed Tai Chi movements was too low to serve as an appropriate training stimulus for healthy people. This could also be the reason why out of nine studies that investigated lower limb strength, only five studies ${ }^{192533} 3637$ found a significant increase. Lan $e t a \tilde{l}^{54}$ previously remarked a relationship between simplified movements and insufficient intensity. This is supported by Wen et als biomechanical analysis of four Tai Chi stances (poses). ${ }^{55}$ They reported a $58.9 \%$ higher knee flexion range of motion and $40.2 \%$ higher peak knee 
extension moment for the bow stance (lunge-position) performed in a 'lower' versus 'higher' stance, where the latter resulted in less knee flexion.

Both, the performance of the SLS test with open eyes and thoracolumbar spine flexibility showed a significant increase in this meta-analysis. According to Kasukawa $e t$ $a \bar{l}^{56}$ and Ishikawa $e t a l{ }^{57}$ there is an interrelation between spinal mobility, sagittal spinal shape and balance impairment, which is caused by displacement of the centre of gravity. Thus, it is not surprising that the study of Zhang et $a l^{46}$ that included balance-impaired participants with a higher risk of falling (defined by one leg standing time between 5 and $20 \mathrm{~s}$ ), reported the greatest improvement in thoracolumbar spine flexibility and also a significant improvement in the SLS test with open eyes performance. A possible correlation between thoracolumbar spine flexibility and balance ability is supported by the result of the univariate meta-regression analysis. Thus, in case of thoracolumbar spine flexibility 91.4\% ( $\mathrm{p}=0.029)$ of the between study heterogeneity could be explained by balance impairment as explanatory variable.

\section{Strengths and limitations}

According to our study design, only studies that used tests applied in health-related fitness or competitive sports contexts were included. Therefore, the strength of our study lies in the fact that the outcomes of these tests were not mixed with clinical assessment tests.

An additional strength, although a disadvantage with respect of the total number of included studies, is that numerous studies that used clinical assessment tests adapted to severely ill individuals, such as the Timed Up and Go test, were excluded. Additionally, studies that did not assign participants randomly to groups were also not considered. For example, one study ${ }^{54}$ that found significant improvements in physical endurance, knee extensor muscle strength and thoracolumbar spine flexibility was excluded. Thus, a relatively small number of studies were eligible for inclusion in the meta-analysis.

The major limitation was the considerable between study heterogeneity that, according to the meta-regression, resulted neither from the explanatory variables different numbers of movements, existing balance impairment (except in the case of thoracolumbar spine flexibility), nor from the presence of a chronic disease. One important reason that could explain the heterogeneity and divergent results among the studies was the vague description of the Tai Chi intervention provided by most of them. Thus, comparison of the quality and intensity of the Tai Chi movements was not possible. Moreover, differences in intervention duration and weekly training frequency, as well as training duration, further hindered comparisons. This is supported by two studies ${ }^{26} 27$ that analysed the effect of the different styles of Tai Chi, and concluded that movements based on the Chen style have superior effects on aerobic capacity, ${ }^{26}$ balance ${ }^{26} 27$ and arterial stiffness. ${ }^{27}$

\section{CONCLUSIONS}

In summary, improvements in handgrip strength (a predictor of overall total muscular strength and functional health) and walking distance, as well as in the related balance ability and thoracolumbar spine flexibility, show that Tai Chi training is useful for healthy older people, as well as for individuals suffering from chronic diseases such as osteoarthritis, COPD or fibromyalgia.

In addition, an increase in postural balance hypothetically derives not only from slow motions of the legs and kicking movements while standing on one leg, but is also positively influenced by the thoracolumbar spine flexibility which is probably supported by the rotational motion of the upper body during the performance of Tai Chi movements.

The slightly increase of handgrip strength, the small number of studies that reported a significant increase of lower limb strength and the decrease in 12MWT walking distance in healthy people suggest the importance of performing high-intensity Tai Chi movements, for example, use of lower stances with greater flexion of the knees. To assess the intensity and quality of the Tai Chi movements performed, photographs would be helpful. For example, Leung $e t a l^{p 7}$ provided a series of photographs that demonstrated the performance of the movements, but such studies are very rare. Further RCTs including younger healthy participants performing a widely used, standardised form (eg, Peking-style routine) of Tai Chi, and practising high-intensity movements (eg, use of lower stances), are needed to evaluate the overall impact of Tai Chi on muscular strength, physical endurance, postural balance and flexibility.

Contributors ChW designed the systematic review and meta-analysis and performed the searches. WS reviewed the search results. Data extraction was performed by ChW and CaW. Data validation was performed by ChW, CaW, CB and WS. Data synthesis was performed by ChW and MA. Meta-analysis was performed by MA. The article was written by ChW, with revisions suggested by CaW, CB, MA and WS.

Funding The authors have not declared a specific grant for this research from any funding agency in the public, commercial or not-for-profit sectors.

Competing interests None declared.

Patient consent for publication Not required.

Ethics approval This systematic review and meta-analysis used pooled data from previously published peer-reviewed articles; ethics approval was obtained by the authors of those studies.

Provenance and peer review Not commissioned; externally peer reviewed.

Open access This is an open access article distributed in accordance with the Creative Commons Attribution Non Commercial (CC BY-NC 4.0) license, which permits others to distribute, remix, adapt, build upon this work non-commercially, and license their derivative works on different terms, provided the original work is properly cited, appropriate credit is given, any changes made indicated, and the use is non-commercial. See: http://creativecommons.org/licenses/by-nc/4.0/.

ORCID iD

Christian Wehner http://orcid.org/0000-0002-2656-8038

\section{REFERENCES}

1 Guo Y, Qiu P, Liu T. Tai Ji Quan: an overview of its history, health benefits, and cultural value. J Sport Health Sci 2014;3:3-8. 
2 Liu HH, Yeh NC, YF W. Effects of Tai Chi Exercise on Reducing Falls and Improving Balance Performance in Parkinson's Disease: A MetaAnalysis. Parkinsons Dis 2019.

3 Huang Z-G, Feng Y-H, Li Y-H, et al. Systematic review and metaanalysis: tai chi for preventing falls in older adults. BMJ Open 2017;7:e013661.

4 Chen Y-W, Hunt MA, Campbell KL, et al. The effect of tai chi on four chronic conditions - cancer, osteoarthritis, heart failure and chronic obstructive pulmonary disease: a systematic review and metaanalyses. Br J Sports Med 2016;50:397-407.

5 Ngai SPC, Jones AYM, Tam WWS, et al. Tai chi for chronic obstructive pulmonary disease (COPD). Cochrane Database Syst Rev 2016;425082:1-71.

6 Zhou Z, Zhou R, Li K, et al. Effects of tai chi on physiology, balance and quality of life in patients with type 2 diabetes: a systematic review and meta-analysis. $J$ Rehabil Med 2019;51:405-17.

7 Liu T, Chan AW, Liu YH, et al. Effects of tai Chi-based cardiac rehabilitation on aerobic endurance, psychosocial well-being, and cardiovascular risk reduction among patients with coronary heart disease: a systematic review and meta-analysis. Eur J Cardiovasc Nurs 2018;17:368-83

8 Zou L, Zhang Y, Yang L, et al. Are mindful exercises safe and beneficial for treating chronic lower back pain? A systematic review and meta-analysis of randomized controlled trials. J Clin Med 2019;8:628-15.

9 Zhang Y, Loprinzi PD, Yang L, et al. The beneficial effects of traditional Chinese exercises for adults with low back pain: a metaanalysis of randomized controlled trials. Medicina 2019;55:118-12.

10 Qin J, Zhang Y, Wu L, et al. Effect of tai chi alone or as additional therapy on low back pain: systematic review and meta-analysis of randomized controlled trials. Medicine 2019;98:e17099.

11 Hempel S, Taylor SL, Solloway M. Evidence Map of Tai Chi. Department of Veterans Affairs, Health Services Research \& Development Service, VA-ESP Project \#ESP 05-226, 2014: 1-37. https://www.ncbi.nlm.nih.gov/books/n/vataichi/pdf/

12 Weineck J. Optimales training: Leistungsphysiologische Trainingslehre unter besonderer Berücksichtigung des Kinder- und Jugendtrainings. Balingen: Spitta, 2002.

13 Li L, Cheng S, Wang G, et al. Tai chi chuan exercises improve functional outcomes and quality of life in patients with primary total knee arthroplasty due to knee osteoarthritis. Complement Ther Clin Pract 2019;35:121-5.

14 Hackney ME, Earhart GM. Tai chi improves balance and mobility in people with Parkinson disease. Gait Posture 2008;28:456-60.

15 You T, Ogawa EF, Thapa S, et al. Effects of tai chi on beta endorphin and inflammatory markers in older adults with chronic pain: an exploratory study. Aging Clin Exp Res 2020;32:1389-92.

16 Council of Europe, Committee for the Development of Sport and UKK Institute for Health Promotion Research. Eurofit for adults. assessment of health-related fitness. Tampere (Finland): Council of Europe Publishing, 1995.

17 Moher D, Liberati A, Tetzlaff J, et al. Preferred reporting items for systematic reviews and meta-analyses: the PRISMA statement. PLoS Med 2009;6:e1000097.

18 Cochrane Collaboration. Data collection form for intervention reviews: RCTs only - version 3, 2014. Available: http://training. cochrane.org/sites/training.cochrane.org/files/public/uploads/ resources/downloadable_resources/English/ERC $\% 20$ data\% 20 collection\%20form\%20for\%20intervention\%20reviews\%20for\% 20RCTs\%20only\%20\%281\%29.doc [Accessed 17 Nov 2017].

19 Audette JF, Jin YS, Newcomer R, et al. Tai chi versus brisk walking in elderly women. Age Ageing 2006;35:388-93.

20 Chyu M-C, James CR, Sawyer SF, et al. Effects of tai chi exercise on posturography, gait, physical function and quality of life in postmenopausal women with osteopaenia: a randomized clinical study. Clin Rehabil 2010;24:1080-90.

21 Liu J, Wang X-Q, Zheng J-J, et al. Effects of tai chi versus proprioception exercise program on neuromuscular function of the ankle in elderly people: a randomized controlled trial. Evid Based Complement Alternat Med 2012;2012:1-8.

22 Zou L, Wang C, Tian Z, et al. Effect of Yang-Style tai chi on gait parameters and musculoskeletal flexibility in healthy Chinese older women. Sports 2017:5:52.

23 Hwang H-F, Chen S-J, Lee-Hsieh J, et al. Effects of home-based tai chi and lower extremity training and Self-Practice on falls and functional outcomes in older Fallers from the emergency Department-A randomized controlled trial. J Am Geriatr Soc 2016:64:518-25.

$24 \mathrm{Ni} \mathrm{M}$, Mooney K, Richards L, et al. Comparative impacts of tai chi, balance training, and a specially-designed yoga program on balance in older fallers. Arch Phys Med Rehabil 2014;95:1620-8.
25 Suksom D, Siripatt A, Lapo P, et al. Effects of two modes of exercise on physical fitness and endothelial function in the elderly: exercise with a flexible stick versus tai chi. J Med Assoc Thai 2011;94:123-32.

26 Zou L, Loprinzi PD, Yu JJ, et al. Superior effects of modified ChenStyle tai chi versus 24-Style tai chi on cognitive function, fitness, and balance performance in adults over 55. Brain Sci 2019;9:102.

27 Zhou S, Zhang Y, Kong Z, et al. The effects of tai chi on markers of atherosclerosis, lower-limb physical function, and cognitive ability in adults aged over 60: a randomized controlled trial. Int $J$ Environ Res Public Health 2019;16:753.

28 Mustian KM, Katula JA, Zhao H. A pilot study to assess the influence of tai chi chuan on functional capacity among breast cancer survivors. J Support Oncol 2006;4:139-45.

29 Sun J, Kanagawa K, Sasaki J, et al. Tai chi improves cognitive and physical function in the elderly: a randomized controlled trial. $J$ Phys Ther Sci 2015;27:1467-71.

30 Wang C. Tai chi improves pain and functional status in adults with rheumatoid arthritis: results of a pilot single-blinded randomized controlled trial. Med Sport Sci 2008;52:218-29.

31 Wolf SL, Barnhart HX, Kutner NG, et al. Selected as the best paper in the 1990s: reducing frailty and falls in older persons: an investigation of tai chi and computerized balance training. J Am Geriatr Soc 2003;51:1794-803.

32 Woo J, Hong A, Lau E, et al. A randomised controlled trial of tai chi and resistance exercise on bone health, muscle strength and balance in community-living elderly people. Age Ageing 2007:36:262-8.

$33 \mathrm{Li} \mathrm{JX}, \mathrm{Xu} \mathrm{DQ}$, Hong Y. Changes in muscle strength, endurance, and reaction of the lower extremities with tai chi intervention. J Biomech 2009:42:967-71.

34 Lu X, Hui-Chan CWY, Tsang WWN. Effects of tai chi training on arterial compliance and muscle strength in female seniors: a randomized clinical trial. Eur J Prev Cardiol 2013;20:238-45.

35 Adler PA. The effects of tai chi on pain and function in older adults with osteoarthritis. Frances Payne Bolton school of nursing. Case Western reserve University, 2007: 1-142. https://etd.ohiolink. edu/letd.send_file?accession=case1168615967\&disposition= attachment

36 Wong A, Figueroa A, Sanchez-Gonzalez MA, et al. Effectiveness of tai chi on cardiac autonomic function and symptomatology in women with fibromyalgia: a randomized controlled trial. J Aging Phys Act 2018;26:214-21.

37 Leung RWM, McKeough ZJ, Peters MJ, et al. Short-Form Sun-style t'ai chi as an exercise training modality in people with COPD. Eur Respir J 2013;41:1051-7.

38 Wang $\mathrm{C}$, Schmid $\mathrm{CH}$, Rones $\mathrm{R}$, et al. A randomized trial of tai chi for fibromyalgia. N Engl J Med 2010;363:743-54.

39 Wang L, Wu K, Chen X, et al. The effects of tai chi on lung function, exercise capacity and health related quality of life for patients with chronic obstructive pulmonary disease: a pilot study. Heart Lung Circ 2019;28:1206-12.

40 Gatts S. Neural mechanisms underlying balance control in tai chi. Med Sport Sci 2008;52:87-103.

41 Hartman CA, Manos TM, Winter C, et al. Effects of T'ai chi training on function and quality of life indicators in older adults with osteoarthritis. J Am Geriatr Soc 2000;48:1553-9.

$42 \mathrm{Li} \mathrm{F}$, Harmer P, Fisher KJ, et al. Tai chi and fall reductions in older adults: a randomized controlled trial. J Gerontol A Biol Sci Med Sci 2005:60:187-94.

43 Li JX, Xu DQ, Hong Y. Effects of 16-week tai chi intervention on postural stability and proprioception of knee and ankle in older people. Age Ageing 2008;37:575-8.

44 Sun W, Wang L, Zhang C, et al. Detraining effects of regular tai chi exercise on postural control ability in older women: a randomized controlled trial. J Exerc Sci Fit 2018;16:55-61.

45 Taylor-Piliae RE, Newell KA, Cherin R, et al. Effects of tai chi and Western exercise on physical and cognitive functioning in healthy community-dwelling older adults. J Aging Phys Act 2010;18:261-79.

46 Zhang J-G, Ishikawa-Takata K, Yamazaki H, et al. The effects of tai chi Chuan on physiological function and fear of falling in the less robust elderly: an intervention study for preventing falls. Arch Gerontol Geriatr 2006;42:107-16.

47 Zheng G, Lan X, Li M, et al. Effectiveness of tai chi on physical and psychological health of college students: results of a randomized controlled trial. PLoS One 2015;10:e0132605.

48 Cochrane Collaboration. Cochrane Handbook for systematic reviews of interventions. version 5.2.0, 2017. Available: https://training. cochrane.org/sites/training.cochrane.org/files/public/uploads/ resources/Handbook5 1/Chapter_10_Handbook_5_2_10.pdf [Accessed May 2019]. 
49 Bohannon RW. Minimal clinically important difference for grip strength: a systematic review. J Phys Ther Sci 2019;31:75-8.

50 Wind AE, Takken T, Helders PJM, et al. Is grip strength a predictor for total muscle strength in healthy children, adolescents, and young adults? Eur J Pediatr 2010;169:281-7.

51 Taekema DG, Gussekloo J, Maier AB, et al. Handgrip strength as a predictor of functional, psychological and social health. A prospective population-based study among the oldest old. Age Ageing 2010;39:331-7.

52 Bohannon RW, Crouch R. Minimal clinically important difference for change in 6-minute walk test distance of adults with pathology: a systematic review. J Eval Clin Pract 2017;23:377-81.
53 Ainsworth BE, Haskell WL, Whitt MC, et al. Compendium of physical activities: an update of activity codes and Met intensities. Med Sci Sports Exerc 2000;32:S498-516.

54 Lan C, Lai JS, Chen SY, et al. 12-Month tai chi training in the elderly: its effect on health fitness. Med Sci Sports Exerc 1998;30:345-51.

55 Wen C, Cao X, Zhang Y, et al. Knee biomechanics of selected kneeunfriendly movement elements in 42-form tai chi. Int $\mathrm{J}$ Perform Anal Sport 2018;18:1050-66.

56 Kasukawa Y, Miyakoshi N, Hongo M, et al. Relationships between falls, spinal curvature, spinal mobility and back extensor strength in elderly people. J Bone Miner Metab 2010;28:82-7.

57 Ishikawa Y, Miyakoshi N, Hongo M, et al. Relationships among spinal mobility and sagittal alignment of spine and lower extremity to quality of life and risk of falls. Gait Posture 2017;53:98-103. 